\title{
Achievable Uplink Rates for Massive MIMO with Coarse Quantization
}

\author{
Christopher Mollén, Junil Choi, Erik G. Larsson and Robert W. Heath
}

Conference Publication

Tweet

N.B.: When citing this work, cite the original article.

Original Publication:

Christopher Mollén, Junil Choi, Erik G. Larsson and Robert W. Heath, Achievable Uplink Rates for Massive MIMO with Coarse Quantization, 2017, International Conference on Acoustics, Speech, and Signal Processing

Copyright: www.ieee.org

Postprint available at: Linköping University Electronic Press

http://urn.kb.se/resolve?urn=urn:nbn:se:liu:diva-135787

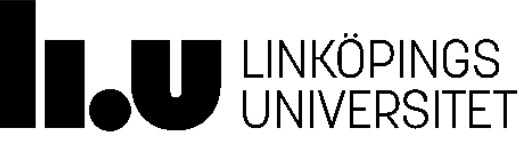




\title{
ACHIEVABLE UPLINK RATES FOR MASSIVE MIMO WITH COARSE QUANTIZATION
}

\author{
Christopher Mollén ${ }^{\mathrm{LU}}$, Junil Choi ${ }^{\mathrm{POSTECH}}$, Erik G. Larsson ${ }^{\mathrm{LU}}$, and Robert W. Heath Jr. ${ }^{\mathrm{UT}}$ \\ ${ }^{\text {Lu }}$ Linköping University, Dept. of Electrical Engineering, 58183 Linköping, Sweden \\ толтесн POSTECH, Dept. of Electrical Engineering, Pohang 37673, South Korea \\ ${ }^{\text {UT }}$ University of Texas at Austin, Dept. of Electrical and Computer Engineering, Austin, TX 78712, USA
}

\begin{abstract}
The high hardware complexity of a massive MIMO base station, which requires hundreds of radio chains, makes it challenging to build commercially. One way to reduce the hardware complexity and power consumption of the receiver is to lower the resolution of the analog-todigital converters (ADCs). We derive an achievable rate for a massive MIMO system with arbitrary quantization and use this rate to show that ADCs with as low as 3 bits can be used without significant performance loss at spectral efficiencies around $3.5 \mathrm{bpcu}$ per user, also under interference from stronger transmitters and with some imperfections in the automatic gain control.
\end{abstract}

Index Terms - ADC, channel estimation, low resolution, massive MIMO, quantization.

\section{INTRODUCTION}

Massive MIMO is a promising technology for the improvement of today's wireless infrastructure [1]. The huge number of transceiver chains required in massive MIMO base stations, however, makes their hardware complexity and cost a challenge that has to be overcome before the technology can become commercially viable [2]. It has been proposed to build each transceiver chain from low-end hardware to reduce the complexity [3].

In this paper, we perform an information theoretical analysis of a massive MIMO system with arbitrary ADCs and derive an achievable rate, which takes quantization into account, for a linear combiner that uses low-complexity channel estimation. The achievable rate is used to draw the conclusion that ADCs with 3 bits are sufficient to achieve a rate close to that of an unquantized system, see Section 6 for more detailed conclusions. This analysis is an extension of work in [4], where we only study one-bit ADCs.

Previous work has studied the capacity of the one-bit quantized frequency-flat MIMO channel [5,6], developed detection and channel estimation methods for the frequency-flat multiuser MIMO channel [7-9] and for the frequency-selective channel [10,11]. Low-resolution ADCs were studied in [12] and the use of a mix of ADCs with different resolutions in [13]. While the methods for frequency-flat channels are hard to extend to frequency-selective channels and the methods for frequency-selective channels either have high computational complexity, require long pilot sequences or imply impractical design changes to the massive MIMO base station, the linear detector and channel estimator that we study is the same low-complexity methods that has been proven possible to implement in practical testbeds $[14,15]$.

A parametric model for hardware imperfections was proposed in [16], where the use of low-resolution ADCs in massive MIMO also was suggested. The parametric model is used in [17] to show that 4-5 bits of resolution maximizes the spectral efficiency for a given power consumption. Several system simulations have been performed to analyze low-resolution ADCs, e.g. [18, 19], where the conclusions coincide with the conclusions in this paper: that three-bit ADCs are sufficient to obtain a performance close to an unquantized system.

\section{SYSTEM MODEL}

The uplink transmission from $K$ single-antenna users to a massive MIMO base station with $M$ antennas is studied. The transmission is based on pulse-amplitude modulation and, for the reception, a matched filter is used for demodulation. It is assumed that the matched filter is implemented as an analog filter and that its output is sampled at symbol rate by an ADC with finite resolution. Because the nonlinear quantization of the ADC comes after the matched filter, the transmission can be studied in symbol-sampled discrete time.

Each user $k$ transmits the signal $\sqrt{P_{k}} x_{k}[n]$, which is normalized,

$$
\mathrm{E}\left[\left|x_{k}[n]\right|^{2}\right]=1,
$$

so that $P_{k}$ denotes the transmit power. The channel from user $k$ to antenna $m$ at the base station is described by its impulse response $\sqrt{\beta_{k}} h_{m k}[\ell]$, which can be factorized into a large-scale fading coefficient $\beta_{k}$ and a small-scale fading impulse response $h_{m k}[\ell]$. The large-scale fading varies slowly in comparison to the symbol rate and it is assumed that it can be accurately estimated with little overhead by both user and base station. How the large-scale fading is estimated with low-resolution ADCs is left for future research. It is therefore assumed to be known throughout the system. The small-scale fading, in contrast, is a priori unknown to everybody. It is independent across $\ell$ and follows the power delay profile

$$
\sigma_{k}^{2}[\ell] \triangleq \mathrm{E}\left[\left|h_{m k}[\ell]\right|^{2}\right],
$$

however, is assumed to be known. It is also assumed that $\sigma_{k}^{2}[\ell]=0$ for all taps $\ell \notin[0, \ldots, L-1]$, where $L$ is the number of nonzero channel taps. Since variations in received power should be described by the large-scale fading only, the power delay profile is normalized such that

$$
\sum_{\ell=0}^{L-1} \sigma_{k}^{2}[\ell]=1, \quad \forall k
$$

Base station antenna $m$ receives the signal

$$
y_{m}[n]=\sum_{k=1}^{K} \sqrt{\beta_{k} P_{k}} \sum_{\ell=0}^{L-1} h_{m k}[\ell] x_{k}[n-\ell]+z_{m}[n] .
$$

The thermal noise of the receiver $z_{m}[n]$ is modeled as a white stochastic process, for which $z_{m}[n] \sim \mathscr{C} \mathcal{N}\left(0, N_{0}\right)$. The received power is denoted

$$
P_{\mathrm{rx}} \triangleq \mathrm{E}\left[\left|y_{m}[n]\right|^{2}\right]=\sum_{k=1}^{K} \beta_{k} P_{k}+N_{0}
$$

Copyright 2017 IEEE. Published in the IEEE 2017 International Conference on Acoustics, Speech, and Signal Processing (ICASSP 2017), scheduled for 5-9 March 2017 in New Orleans, Louisiana, USA. Personal use of this material is permitted. However, permission to reprint/republish this material for advertising or promotional purposes or for creating new collective works for resale or redistribution to servers or lists, or to reuse any copyrighted component of this work in other works, must be obtained from the IEEE. Contact: Manager, Copyrights and Permissions / IEEE Service Center / 445 Hoes Lane / P.O. Box 1331 / Piscataway, NJ 08855-1331, USA. Telephone: + Intl. 908-562-3966. 
Transmission is assumed to be done with a cyclic prefix in blocks of $N$ symbols. The received signal can than be given in the frequency domain as

$$
y_{m}[v] \triangleq \frac{1}{\sqrt{N}} \sum_{n=0}^{N-1} y_{m}[n] e^{-j 2 \pi n v / N}=\sum_{k=1}^{K} h_{m k}[v] x_{k}[v]+z_{m}[v],
$$

The Fourier transforms $x_{k}[v]$ and $z_{k}[v]$ of the transmit signal $x_{k}[n]$ and noise $z_{m}[n]$ are defined in the same way as $y_{m}[v]$. The frequency response of the channel is defined as

$$
h_{m k}[v] \triangleq \sum_{\ell=0}^{L-1} h_{m k}[\ell] e^{-j 2 \pi \ell v / N} .
$$

\section{QUANTIZATION}

The inphase and quadrature signals are assumed to be quantized separately by two identical ADCs with quantization levels given by $\mathcal{Q}_{\mathrm{re}_{\mathrm{e}}} \subseteq$ $\mathbb{R}$. The set of quantization points is denoted $Q \triangleq\left\{a+j b: a, b \in \mathcal{Q}_{\mathfrak{R e}_{\mathrm{e}}}\right\}$ and the quantization by

$$
[y]_{\mathscr{Q}} \triangleq \underset{q \in \mathscr{Q}}{\arg \min }|y-q| .
$$

To adjust the input signal to the dynamic range of the ADC, an automatic gain control scales the input power by $A$. The ADC outputs:

$$
q_{m}[n] \triangleq\left[\sqrt{A} y_{m}[n]\right]_{\mathscr{Q}} .
$$

Using the orthogonality principle, any signal with finite power can be partitioned into one part $\rho y_{m}[n]$ that is correlated to the transmit signal and one part $e_{m}[n]$ that is uncorrelated:

$$
q_{m}[n]=\rho y_{m}[n]+e_{m}[n] .
$$

The constant $\rho$ and the variance of the uncorrelated part are given by:

$$
\begin{aligned}
\rho & =\frac{\mathrm{E}\left[q_{m}[n] y_{m}^{*}[n]\right]}{\mathrm{E}\left[\left|y_{m}[n]\right|^{2}\right]}, \\
\mathrm{E}\left[\left|e_{m}[n]\right|^{2}\right] & =\mathrm{E}\left[\left|q_{m}[n]\right|^{2}\right]-\frac{\left|\mathrm{E}\left[q_{m}[n] y_{m}^{*}[n]\right]\right|^{2}}{\mathrm{E}\left[\left|y_{m}[n]\right|^{2}\right]} .
\end{aligned}
$$

The normalized mean-square error (MSE) of the quantization is denoted by:

$$
\begin{aligned}
Q & \triangleq \frac{1}{|\rho|^{2}} \mathrm{E}\left[\left|e_{m}[n]\right|^{2}\right] \\
& =P_{\mathrm{rx}}\left(\frac{\mathrm{E}\left[\left|q_{m}[n]\right|^{2}\right] \mathrm{E}\left[\left|y_{m}[n]\right|^{2}\right]}{\left|\mathrm{E}\left[q_{m}[n] y_{m}^{*}[n]\right]\right|^{2}}-1\right) .
\end{aligned}
$$

An ADC with $b$-bit resolution has $\left|\mathcal{Q}_{\text {re }}\right|=2^{b}$ quantization levels. In [20], the quantization levels that minimize the MSE for a Gaussian input signal with unit variance are derived numerically for $1-5$ bit ADCs, both with arbitrarily and uniformly spaced quantization levels. The normalized MSE of the quantization has been computed numerically and is given in Table 1 for the optimized quantizers. To obtain the MSE in Table 1 with the quantization levels from [20], the input should be a unit-variance Gaussian signal and the automatic gain control $A=A^{\star} \triangleq 1 / P_{\mathrm{rx}}$. Figure 1 shows how the quantization MSE in a four-bit ADC changes with imperfect gain control. Even if the gain control varies between -8 and $5 \mathrm{~dB}$ from the optimal value, the MSE is still better than that of a three-bit ADC.
Table 1: Normalized quantization mean square-error $Q / P_{\mathrm{rx}}$ \begin{tabular}{l|lllll} 
resolution $b$ & 1 & 2 & 3 & 4 & 5 \\
\hline optimal levels & 0.5708 & 0.1331 & 0.03576 & 0.009573 & 0.002492 \\
uniform levels & 0.5708 & 0.1349 & 0.03889 & 0.01166 & 0.003506
\end{tabular}

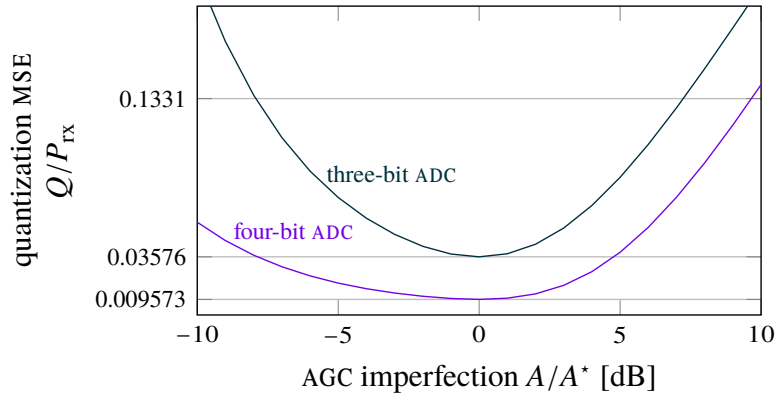

Fig. 1: Quantization MSE for optimal four-bit ADC with imperfect AGC.

\section{CHANNEL ESTIMATION}

Channel estimation is done by receiving $N=N_{\mathrm{p}}$-symbol long orthogonal pilots from the users, i.e., pilots $x_{k}[n]$ such that:

$$
\sum_{n=0}^{N_{\mathrm{p}}-1} x_{k}[n] x_{k^{\prime}}^{*}[n+\ell]=\left\{\begin{array}{ll}
N_{\mathrm{p}}, & \text { if } k=k^{\prime}, \ell=0 \\
0, & \text { if } k \neq k^{\prime}, \ell=1, \ldots, L-1
\end{array},\right.
$$

where the indices are taken modulo $N_{\mathrm{p}}$. To fulfill (15), $N_{\mathrm{p}} \geq K L$. We will call the factor of extra pilots $\mu \triangleq N_{\mathrm{p}} /(K L)$ the pilot excess factor. As remarked upon in [4], not all sequences fulfilling (15) result in the same performance. Here we use the pilots proposed in [4]. Using (10) and (15), an observation of the channel is obtained by correlation:

$$
\begin{aligned}
r_{m k}[\ell] & =\frac{1}{\rho \sqrt{N_{\mathrm{p}}}} \sum_{n=0}^{N_{\mathrm{p}}-1} q_{m}[n] x_{k}^{*}[n+\ell] \\
& =\sqrt{\beta_{k} P_{k} N_{\mathrm{p}}} h_{m k}[\ell]+e_{m k}^{\prime}[\ell]+z_{m k}^{\prime}[\ell],
\end{aligned}
$$

where

$$
\begin{aligned}
& e_{m k}^{\prime}[\ell] \triangleq \frac{1}{\rho \sqrt{N_{\mathrm{p}}}} \sum_{n=0}^{N_{\mathrm{p}}-1} e_{m}[n] x_{k}^{*}[n+\ell], \\
& z_{m k}^{\prime}[\ell] \triangleq \frac{1}{\sqrt{N_{\mathrm{p}}}} \sum_{n=0}^{N_{\mathrm{p}}-1} z_{m}[n] x_{k}^{*}[n+\ell] \sim \mathscr{C} \mathcal{N}\left(0, N_{0}\right) .
\end{aligned}
$$

The linear minimum MSE estimate of the frequency response of the channel is thus

$$
\hat{h}_{m k}[v]=\sum_{\ell=0}^{L-1} \frac{\sqrt{\beta_{k} P_{k} N_{\mathrm{p}}} \sigma_{k}^{2}[\ell]}{\beta_{k} P_{k} N_{\mathrm{p}} \sigma_{k}^{2}[\ell]+Q+N_{0}} r_{m k}[\ell] e^{-j 2 \pi \ell v / N}
$$

and the error $\epsilon_{m k}[v] \triangleq \hat{h}_{m k}[v]-h_{m k}[v]$ has the variance $1-c_{k}$, where the variance of the channel estimate is given by

$$
c_{k} \triangleq \mathrm{E}\left[\left|\hat{h}_{m k}[v]\right|^{2}\right]=\sum_{\ell=0}^{L-1} \frac{\sigma_{k}^{4}[\ell] \beta_{k} P_{k} N_{\mathrm{p}}}{\sigma_{k}^{2}[\ell] \beta_{k} P_{k} N_{\mathrm{p}}+Q+N_{0}} .
$$

Figure 2 shows the variance of the channel estimate. A resolution of 2 bit is enough to obtain a channel estimate that is only $0.5 \mathrm{~dB}$ worse than in an unquantized system. With a resolution of 3 bit or higher, the variance of the channel estimate is practically the same as that 

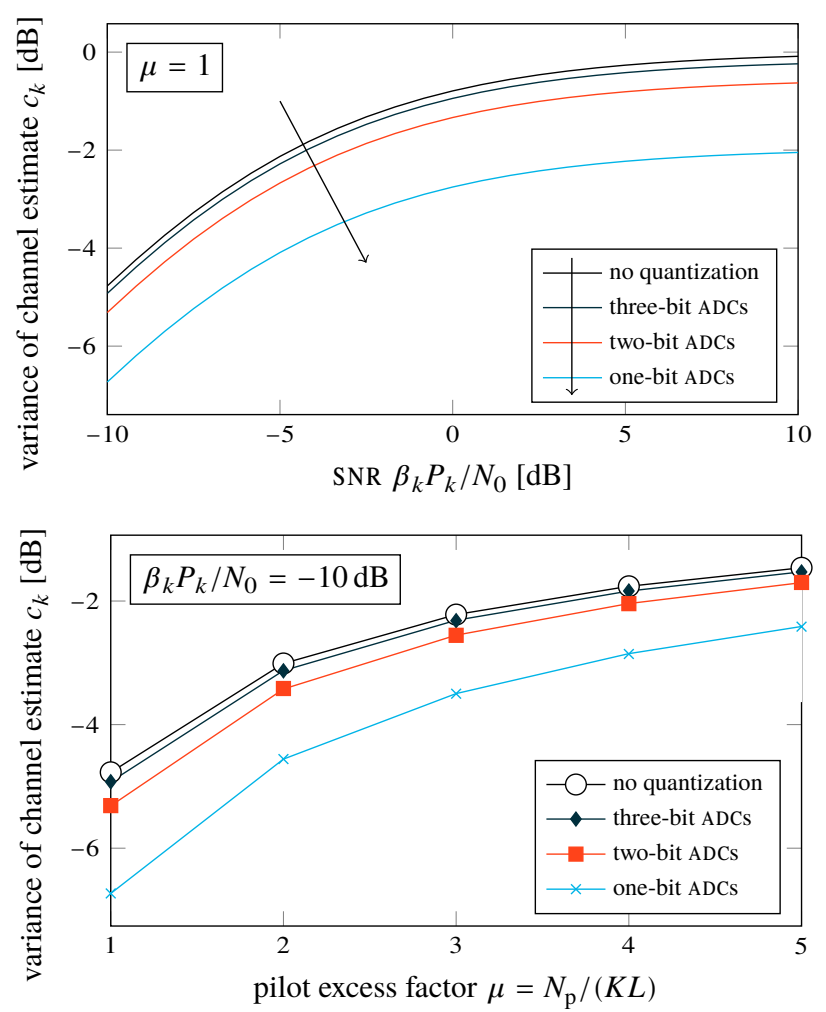

Fig. 2: The quality of the channel estimate with 5 users and a uniform power delay profile $\sigma_{k}^{2}[\ell]=1 / L$, for all $k, \ell$, and with equal received power $\beta_{k} P_{k}=\beta_{1} P_{1}$ from all users $k$. The optimal quantization levels derived in [20] are used. The markers show how simulated results with $L=20$ channel taps corroborate the theory. When $c_{k}=0 \mathrm{~dB}$, perfect channel state information is obtained.

of the unquantized system. Increasing the pilot length, increases the quality of the channel estimation in all systems. The improvement is, however, the largest when going from $\mu=1$ to $\mu=2$; thereafter the improvement gets smaller.

\section{DATA TRANSMISSION}

The uplink data is transmitted in a block of length $N=N_{\mathrm{u}}$, which is separated from the pilot block in time. The received signal is processed in a linear combiner and an estimate of the transmitted signal is obtained by

$$
\hat{x}_{k}[v]=\frac{1}{\rho} \sum_{m=1}^{M} w_{m k}[v] q_{m}[v],
$$

where the Fourier transform $q_{m}[v]$ of $q_{m}[n]$ is defined in the same way as $y_{m}[v]$ in (6) and the combiner weights $w_{m k}[v]$ are chosen as a function of the channel estimate. For example, the maximum-ratio and zero-forcing combiners can be used, see [4].

If we code over many channel realizations, an achievable ergodic rate, i.e. lower bound on the channel capacity, is given by [4], independent of $v$ :

$$
R_{k}=\log _{2}\left(1+\frac{\left|\mathrm{E}\left[\hat{x}_{k}^{*}[v] x_{k}[v]\right]\right|^{2}}{\mathrm{E}\left[\left|\hat{x}_{k}[v]\right|^{2}\right]-\left|\mathrm{E}\left[\hat{x}_{k}^{*}[v] x_{k}[v]\right]\right|^{2}}\right) .
$$

To compute the expected values in (23), the estimate of the transmit signal in (22) can be expanded by using the relation in (10) and writing

$$
\begin{aligned}
& \text { the channel as } h_{m k}[v]=\hat{h}_{m k}[v]-\epsilon_{m k}[v] \text { : } \\
& \begin{aligned}
\hat{x}_{k}[v]= & x_{k}[v] \sqrt{\beta_{k} P_{k}} \sum_{m=1}^{M} \mathrm{E}\left[w_{m k}[v] \hat{h}_{m k}[v]\right] \\
& +x_{k}[v] \sqrt{\beta_{k} P_{k}} \sum_{m=1}^{M}\left(w_{m k}[v] \hat{h}_{m k}[v]-\mathrm{E}\left[w_{m k}[v] \hat{h}_{m k}[v]\right]\right) \\
& +\sum_{k^{\prime} \neq k} x_{k^{\prime}}[v] \sqrt{\beta_{k^{\prime}} P_{k^{\prime}}} \sum_{m=1}^{M} w_{m k}[v] \hat{h}_{m k^{\prime}}[v] \\
& -\sum_{k^{\prime}=1}^{K} x_{k^{\prime}}[v] \sqrt{\beta_{k^{\prime}} P_{k^{\prime}}} \sum_{m=1}^{M} w_{m k}[v] \epsilon_{m k}[v] \\
& +\sum_{m=1}^{M} w_{m k}[v] z_{m}[v]+\frac{1}{\rho} \sum_{m=1}^{M} w_{m k}[v] e_{m}[v],
\end{aligned}
\end{aligned}
$$

where the Fourier transform $e_{m}[v]$ of $e_{m}[n]$ is defined as in (6). Note that only the first term is correlated to the desired signal. By assuming that the channel is i.i.d. Rayleigh fading, it can be shown that the other terms in (24) — channel gain uncertainty, interference, channel estimation error, thermal noise, quantization error - are mutually uncorrelated and the variance of each term can be evaluated. In [4], it is shown, for one-bit ADCs, that, in limit, the last term does not combine coherently and its variance equals

$$
\mathrm{E}\left[\left|\frac{1}{\rho} \sum_{m=1}^{M} w_{m k}[v] e_{m}[v]\right|^{2}\right] \rightarrow Q, \quad L \rightarrow \infty
$$

if the combiner is normalized such that $\sum_{m=1}^{M} \mathrm{E}\left[\left|w_{m k}[v]\right|^{2}\right]=1$, which will be assumed here. This can be generalized to any quantization in a similar way. The rate in (23) can then be written as

$$
R_{k} \rightarrow \log _{2}\left(1+\frac{\beta_{k} P_{k} c_{k} G}{\sum_{k^{\prime}=1}^{K} \beta_{k^{\prime}} P_{k^{\prime}}\left(1-c_{k^{\prime}}(1-I)\right)+Q+N_{0}}\right),
$$

as $L \rightarrow \infty$, where the array gain and interference terms are defined as

$$
\begin{aligned}
G & \triangleq\left|\sum_{m=1}^{M} \mathrm{E}\left[w_{m k}[v] \hat{h}_{m k}[v]\right]\right|^{2}, \\
I & \triangleq \operatorname{Var}\left(\sum_{m=1}^{M} w_{m k}[v] \hat{h}_{m k^{\prime}}[v]\right),
\end{aligned}
$$

where

$$
G=\left\{\begin{array}{l}
M \\
M-K
\end{array}, \quad I=\left\{\begin{array}{ll}
1, & \text { for maximum-ratio combining } \\
0, & \text { for zero-forcing combining }
\end{array} .\right.\right.
$$

Only the quantization error in (25) depends on the number of channel taps $L$. In the unquantized case, $R_{k}$ thus does not depend on $L$. In other cases, the dependency on $L$ vanishes as $L \rightarrow \infty$ and the energy of the received signal no longer depends on the energy of the detected symbol [4, Lemmata 1,3]; this argument is valid for any quantizer.

The dependency on $L$ quickly becomes negligible, and the limit in (26) can approximate the rate with negligible error, also for practical delay spreads $L$. The approximation can even be good for some frequency-flat channels $(L=1)$ when the received power $\sum_{k=1}^{K} \beta_{k} P_{k}$ is small relative to the noise power $N_{0}$ or when the number of users is large and there is no dominant user, i.e., no user $k$ for which $\beta_{k} P_{k} \gg$ $\sum_{k^{\prime} \neq k} \beta_{k^{\prime}} P_{k^{\prime}}$. For general frequency-flat channels, however, it is not true that the variance of the quantization error vanishes with increasing number of antennas, as it does in the limit $L \rightarrow \infty$ in (26); this seems to be overlooked in some of the literature [21-24].

The rate $R_{k}$ is plotted in Figure 3 for maximum-ratio and zeroforcing combining. The transmit powers are allocated proportionally 
(a) Maximum-ratio combining

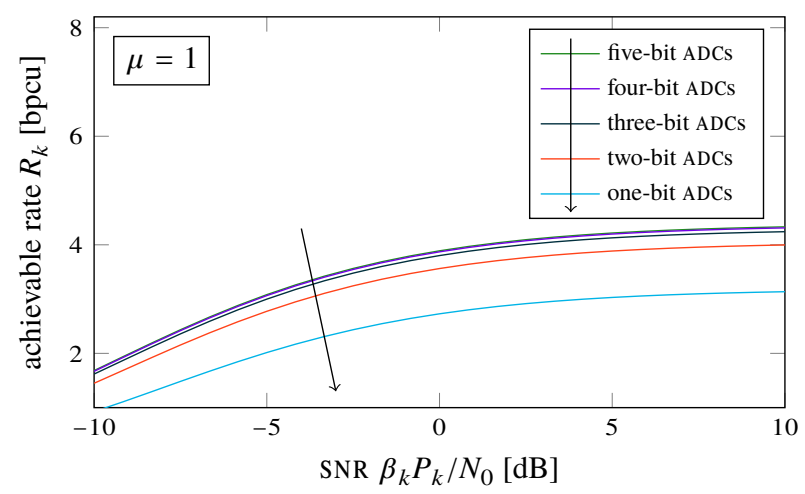

(b) Zero-forcing combining

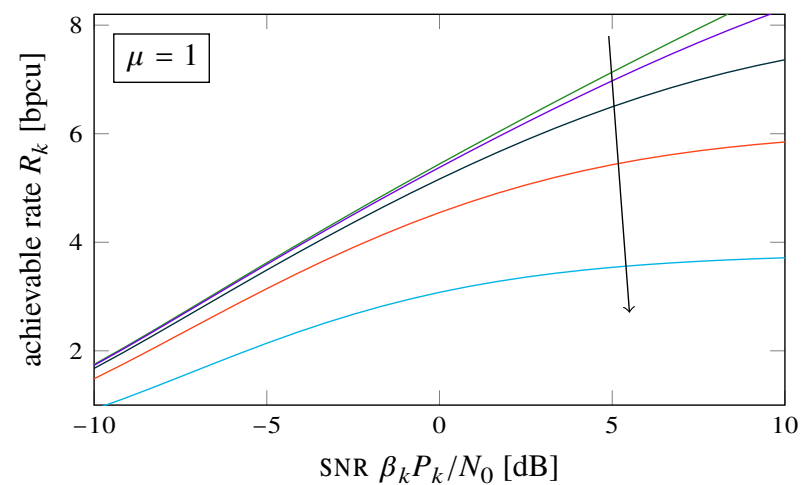

Fig. 3: Rate of a system with 100 antennas and 10 users, where the power is proportional to $1 / \beta_{k}$ and training is done with $N_{\mathrm{p}}=K L$ pilots. The channel is i.i.d. Rayleigh fading with uniform power delay profile $h_{m k}[\ell] \sim \mathscr{C} \mathcal{N}(0,1 / L)$. The optimal quantization levels derived in [20] are used.

to $1 / \beta_{k}$ and channel estimation is done with $N_{\mathrm{p}}=K L$ pilots, i.e., the pilot excess factor $\mu=1$. It can be seen that low-resolution ADCs cause very little performance degradation at spectral efficiencies below 4 bpcu. One-bit ADCs deliver approximately $40 \%$ lower rates than the equivalent unquantized system and the performance degradation becomes practically negligible with ADCs with as few as 3 bit resolution. Assuming that the power dissipation in an ADC is proportional to $2^{b}$, the use of one-bit ADCs thus reduces the ADC power consumption by approximately $6 \mathrm{~dB}$ at the price of $40 \%$ performance degradation compared to the use of three-bit ADCs, which deliver almost all the performance of an unquantized system.

In [18], it is pointed out that low-resolution ADCs create a near-far problem, where users with relatively weak received power drown in the interference from stronger users. This is illustrated with a zero-forcing combiner in Figure 4, where it can be seen how the performance of the weak users degrades if there is a stronger user in the system. Note that the performance degrades also in the unquantized system, where the imperfect channel estimates prevent perfect suppression of the interference from the strong user. In the quantized systems, there is a second cause of the performance degradation: With quantization, the pilots are no longer perfectly orthogonal and the quality of the channel estimates is negatively affected by interference from the strong user. This effect can be seen in (21), where $Q$ scales with the received power $P_{\mathrm{rx}}$ and thus with the power of the interferer.

Figure 4, however, shows that the near-far problem does not become prominent until the received power from the strong user is

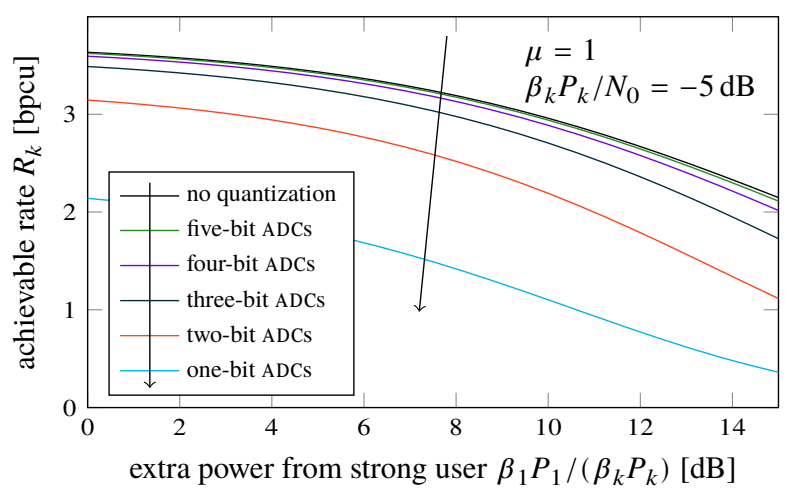

Fig. 4: Per-user rate $R_{k}$ for users $k=2, \ldots, K$ when user $k=1$ has a different receive SNR. The system has 100 antennas and $K=10$ users, the channel is i.i.d. Rayleigh with uniform power delay profile and is estimated with $N_{\mathrm{p}}=K L$ pilots. The optimal quantization levels derived in [20] are used.

around $10 \mathrm{~dB}$ higher than that of the weak users, where the data rate is degraded by approximately $15 \%$ in the unquantized system. The degradation is larger in the quantized systems but the additional degradation due to quantization is almost negligible when the resolution is 3 bits or higher. With one-bit ADCs and one strong user with $10 \mathrm{~dB}$ larger received power, the degradation of the data rate increases to almost $50 \%$. Proper power control among users, however, can eliminate the near-far problem altogether.

\section{CONCLUSION}

We have derived an achievable rate for a single-cell massive MIMO system that takes quantization into account. The derived rate shows that ADCs with as low resolution as 3 bits can be used with negligible performance loss compared to an unquantized system, also with interference from stronger users. For example, with three-bit ADCs, the data rate is decreased by $4 \%$ at spectral efficiencies of $3.5 \mathrm{bpcu}$ in a system with 100 antennas that serves 10 users. It also shows that four-bit ADCs can be used to accommodate for imperfect automatic gain control-imperfections up to $5 \mathrm{~dB}$ still result in better performance than the three-bit ADCs. One-bit ADCs can be built from a single comparator and do not need a complex gain control (which ADCs with more than one-bit resolution need), which simplifies the hardware design of the base station receiver and reduce its power consumption. The derived rate, however, shows that one-bit ADCs lead to a significant rate reduction. For example, one-bit ADCs lead to a $40 \%$ rate reduction in a system with 100 antennas that serves 10 users at spectral efficiencies of $3.5 \mathrm{bpcu}$. In the light of the good performance of three-bit ADCs, whose power consumption should already be small in comparison to other hardware components, the primary reason for the use of one-bit ADCs would be the simplified hardware design, not the lower power consumption.

\section{ACKNOWLEDGMENTS}

The research leading to these results has received funding from the European Union Seventh Framework Programme under grant agreement number ICT-619086 (MAMMOET), the Swedish Research Council (Vetenskapsrådet), the National Science Foundation under grant number NSF-CCF-1527079, and the ICT R\&D program of MSIP/IITP [2016 (B0717-16-0002)]. 


\section{REFERENCES}

[1] E. G. Larsson, O. Edfors, F. Tufvesson, and T. L. Marzetta, "Massive MIMO for next generation wireless systems," IEEE Commun. Mag., vol. 52, no. 2, pp. 186-195, Feb. 2014.

[2] F. Athley, G. Durisi, and U. Gustavsson, "Analysis of massive MIMO with hardware impairments and different channel models," in Proc. Eur. Conf. Antennas Propag. IEEE, Apr. 2015, pp. 1-5.

[3] E. Björnson, J. Hoydis, M. Kountouris, and M. Debbah, "Massive MIMO systems with non-ideal hardware: Energy efficiency, estimation, and capacity limits," IEEE Trans. Inf. Theory, vol. 60, no. 11, pp. 7112-7139, Nov. 2014.

[4] C. Mollén, J. Choi, E. G. Larsson, and R. W. Heath, Jr., "Uplink performance of wideband massive MIMO with one-bit ADCs," IEEE Trans. Wireless Commun., Oct. 2016.

[5] A. Mezghani and J. A. Nossek, "On ultra-wideband MIMO systems with 1-bit quantized outputs: Performance analysis and input optimization," in Proc. IEEE Int. Symp. Inform. Theory, Jun. 2007, pp. 1286-1289.

[6] J. Mo and R. W. Heath, Jr., "Capacity analysis of one-bit quantized MIMO systems with transmitter channel state information," IEEE Trans. Signal Process., vol. 63, no. 20, pp. 5498-5512, Oct. 2015.

[7] M. T. Ivrlač and J. A. Nossek, "On MIMO channel estimation with single-bit signal-quantization," in Proc. ITG Workshop Smart Antennas, 2007.

[8] J. Choi, J. Mo, and R. W. Heath, Jr., "Near maximum-likelihood detector and channel estimator for uplink multiuser massive MIMO systems with one-bit ADCs," IEEE Trans. Commun., vol. 64, no. 5, pp. 2005-2018, Mar. 2016.

[9] C. Risi, D. Persson, and E. G. Larsson, "Massive MIMO with 1-bit ADC," ArXiv E-Print, Apr. 2014, arXiv:1404.7736 [cs.IT].

[10] O. Dabeer and U. Madhow, "Channel estimation with lowprecision analog-to-digital conversion," in Proc. IEEE Int. Conf. Commun., May 2010, pp. 1-6.

[11] S. Wang, Y. Li, and J. Wang, "Multiuser detection in massive spatial modulation MIMO with low-resolution ADCs," IEEE Trans. Wireless Commun., vol. 14, no. 4, pp. 2156-2168, Apr. 2015.

[12] C. Studer and G. Durisi, "Quantized massive MU-MIMOOFDM uplink," IEEE Trans. Commun., vol. 64, no. 6, pp. 2387-2399, Jun. 2016.

[13] N. Liang and W. Zhang, "Mixed-ADC massive MIMO," IEEE J. Sel. Areas Commun., vol. 34, no. 4, pp. 983-997, Apr. 2016.

[14] C. Shepard, H. Yu, N. Anand, E. Li, T. Marzetta, R. Yang, and L. Zhong, "Argos: Practical many-antenna base stations," in Proc. Annu. Int. Conf. Mobile Computing and Networking. ACM, Aug. 2012, pp. 53-64.

[15] J. Vieira, S. Malkowsky, K. Nieman, Z. Miers, N. Kundargi, L. Liu, I. Wong, V. Öwall, O. Edfors, and F. Tufvesson, "A flexible 100-antenna testbed for massive MIMO," in Proc. IEEE Global Commun. Conf., Dec. 2014, pp. 287-293.

[16] E. Björnson, M. Matthaiou, and M. Debbah, "Massive MIMO with non-ideal arbitrary arrays: Hardware scaling laws and circuit-aware design," IEEE Trans. Wireless Commun., vol. 14, no. 8, pp. 4353-4368, Aug. 2015.
[17] D. Verenzuela, E. Björnson, and M. Matthaiou, "Hardware design and optimal ADC resolution for uplink massive MIMO systems," in Proc. Sensor Array and Multichannel Signal Process. Workshop, Jul. 2016.

[18] S. Jacobsson, G. Durisi, M. Coldrey, U. Gustavsson, and C. Studer, "Massive MIMO with low-resolution ADCs," ArXiv E-Print, Feb. 2016, arXiv:1602.01139 [cs.IT].

[19] C. Desset and L. Van der Perre, "Validation of low-accuracy quantization in massive MIMO and constellation EVM analysis," in Proc. Eur. Conf. Networks and Commun., Jun. 2015, pp. $21-25$.

[20] J. Max, "Quantizing for minimum distortion," IRE Trans. Inf. Theory, vol. 6, no. 1, pp. 7-12, Mar. 1960.

[21] L. Fan, S. Jin, C.-K. Wen, and H. Zhang, "Uplink achievable rate for massive MIMO with low-resolution ADC," IEEE Commun. Lett., vol. 19, no. 12, pp. 2186-2189, Dec. 2015.

[22] J. Zhang, L. Dai, S. Sun, and Z. Wang, "On the spectral efficiency of massive MIMO systems with low-resolution ADCs," IEEE Commun. Lett., vol. 20, no. 5, pp. 842-845, May 2016.

[23] Y. Li, C. Tao, L. Liu, G. Seco-Granados, and A. L. Swindlehurst, "Channel estimation and uplink achievable rates in one-bit massive MIMO systems," in Proc. Sensor Array and Multichannel Signal Process. Workshop, Jul. 2016.

[24] Y. Li, C. Tao, L. Liu, A. Mezghani, and A. L. Swindlehurst, "How much training is needed in one-bit massive MIMO systems at low SNR?" ArXiv E-Print, Aug. 2016, arXiv:1608.05468 [cs.IT]. 